\title{
Combination of the DVZ Method, Particle Filter, and Fuzzy Controller for Electric Wheelchair Navigation
}

\author{
Malek Njah and Mohamed Jallouli \\ Control and Energy Management Laboratory (CEMLab), National School of Engineering of Sfax, University of Sfax, BP W, \\ 3038 Sfax, Tunisia
}

Correspondence should be addressed to Malek Njah; njahmalek@yahoo.fr

Received 30 July 2014; Revised 31 October 2014; Accepted 3 November 2014; Published 18 November 2014

Academic Editor: Sandro M. Radicella

Copyright (C) 2014 M. Njah and M. Jallouli. This is an open access article distributed under the Creative Commons Attribution License, which permits unrestricted use, distribution, and reproduction in any medium, provided the original work is properly cited.

\begin{abstract}
Electric wheelchair is one of the many engines used for the movement of aged and disabled people. This paper introduces an obstacle avoidance using deformable virtual zone (DVZ), particle filter to improve localization and fuzzy controller to join desired target. This controller is developed to increase the independence of disabled and aged people, specifically those who suffer not only disability in the lower limbs but also visual disturbances. To overcome these problems, different perceptive abilities or sensors were introduced. In this context, we developed a control system composed by fuzzy controller to join a target, DVZ method for obstacle avoidance, and particle filter for localization. Also, we present the simulation results of the wheelchair navigation system.
\end{abstract}

\section{Introduction}

Several works have been developed in this context. The publication of Lapierre et al. [1] threatened the DVZ method combined with the path followed, and the work of Gil Pinto et al. $[2,3]$ provides an active approach to planning based on the DVZ method. This control strategy is based on the GPS sensor and ultrasonic sensors for generating an optimal trajectory to ensure cooperation between nonholonomic robots in an unknown environment. The particulate filter is a probabilistic method allowing the multisensor data fusion and localization. The coordination of these strategies results in an automatic obstacle avoidance navigation to reach a target point $[4,5]$. This paper presents control system based on the DVZ method for obstacle avoidance. The principle of this method is to define the robot/environment interaction as a risk zone surrounding the wheelchair (Figure 2). The DVZ characterizes the deformable zone geometry and depends on the wheelchair speed. When the risk zone is disturbed by an obstacle, it can be reformed by modifying the velocities of the wheelchair $[1,6]$.

In addition we will detail the use of the particle filter method for localization. This method uses a probabilistic approach $[4,7]$. It generates a set of possible positions in each sampling period. This data fusion method gives the estimated position of the chair using distance between wheelchair and obstacles.

Also, we use a fuzzy controller already developed in previous work that allows the wheelchair to reach the desired position $[8,9]$.

In the following we will detail the used methods and the simulation of the control system.

\section{Using Fuzzy Controller for Electric Wheelchair to Join Target}

In this paper the fuzzy controller permits reaching the target position $\left(X_{T}, Y_{T}\right)$ from any position $(X, Y)$.

The considered wheelchair is composed of two independent coaxial driving wheels and two free-rotating wheels. The configuration of this type of robot is characterized by the position on the $X$-axis, $Y$-axis, and orientation $\theta$ in a Cartesian coordinate system illustrated by Figure 1. Consider the following. 


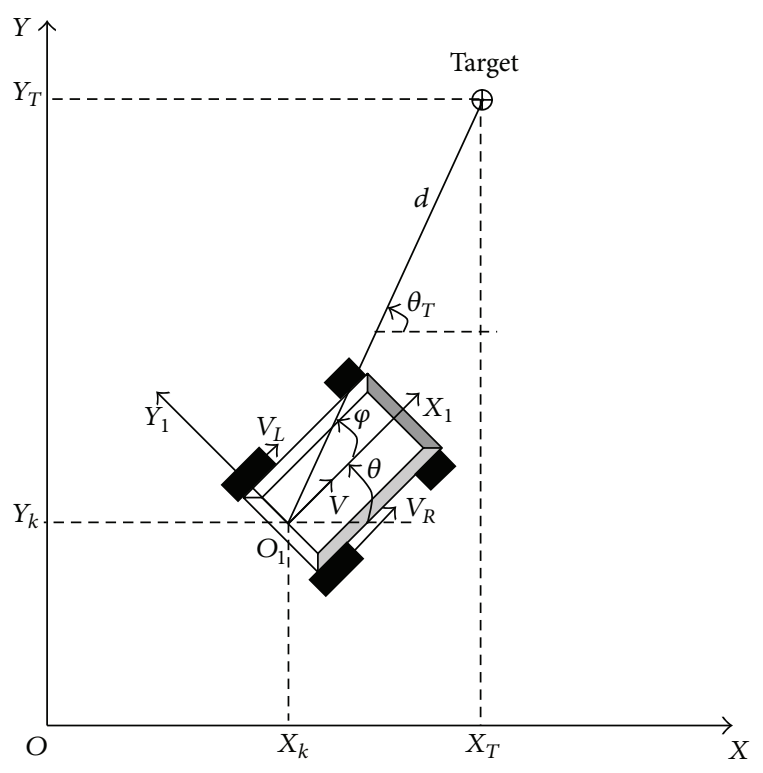

FIGURE 1: Wheelchair and target position.

$\left(X_{k}, Y_{k}\right)$ is wheelchair position at the sampling period $k$.

$V_{L}$ and $V_{R}$ are the velocities of right and left wheels. The objective is to move the electric wheelchair and reach the desired position.

$V$ is linear speed of the electric wheelchair.

$\theta$ is the angle of wheelchair orientation.

$L$ is the distance separating the driving wheels of electric wheelchair.

The distance between the electric wheelchair and the target is defined by the following expression:

$$
\begin{aligned}
& d=\sqrt{\left(X_{T}-X\right)^{2}+\left(Y_{T}-Y\right)^{2}}, \\
& \varphi=\theta_{T}-\theta,
\end{aligned}
$$

where

$$
\theta_{T}=\tan ^{-1} \frac{\left(Y_{T}-Y_{k}\right)}{\left(X_{T}-X_{k}\right)} .
$$

The electric wheelchair is represented with the discrete form of the kinematic model:

$$
\begin{gathered}
X_{(k+1)}=X_{(k)}+T \frac{V_{R(k)}+V_{L(k)}}{2} \cos \theta_{k}, \\
Y_{(k+1)}=Y_{(k)}+T \frac{V_{R(k)}+V_{L(k)}}{2} \sin \theta_{k}, \\
\theta_{(k+1)}=\theta_{(k)}+T \frac{V_{R(k)}-V_{L(k)}}{L},
\end{gathered}
$$

where $T$ is the sampling period.

The used fuzzy controller is developed in our previous work $[8,9]$. When we use the fuzzy controller of the type

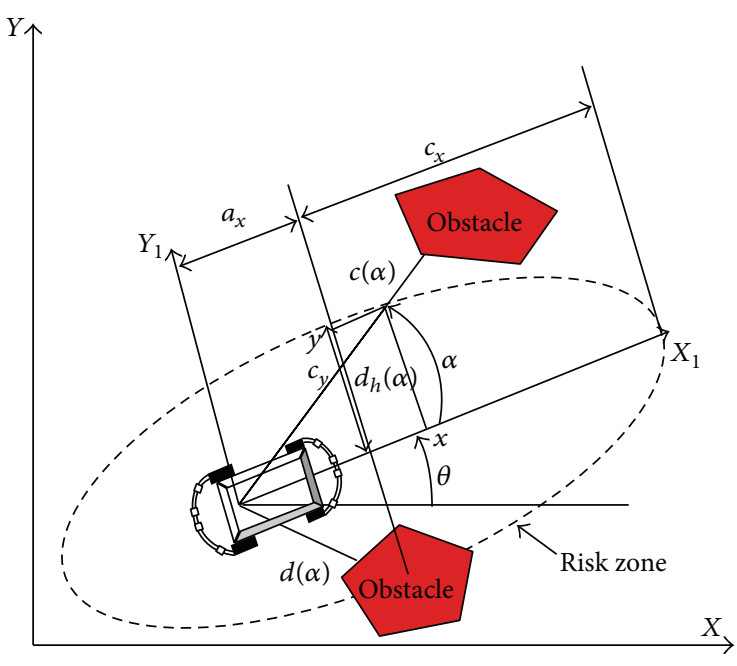

FIGURE 2: Obstacle avoidance with DVZ method.

Takagi-Sugeno of the order 0 , the distance " $d$ " and the angle " $\varphi$ " are the input. The left and the right velocity $\left(V_{L}, V_{R}\right)$ of two driving wheels are the output. The input membership of variables " $d$ " and " $\varphi$," linguistic inference table of velocity, and fuzzy repartition of output variables are detailed in previous papers $[8,9]$. In this paper we combined the fuzzy controller to join defined target with obstacle avoidance using DVZ. We improved the localization using the particle filter. In the next section we describe the DVZ method for obstacle avoidance.

\section{Obstacle Avoidance by the DVZ Method}

The DVZ method, based on a reflex behavior, uses the concept of deformable virtual zone developed by Zapata et al. $[10,11]$. This method associates a risk zone to the electric wheelchair. This zone depends on the kinematics of the wheelchair. The deformation of the risk zone is due to intrusion of proximity information (obstacles within the risk zone). The system response is made to reform the danger zone to its nominal shape. The movement of the platform depends on the area surrounding the robot. If an obstacle is detected, the area will deform. This method treats all forms of obstacles. We can apply this type of control with the use of the kinematic model of the robot, which facilitates its application to a real system that navigates in an unknown environment $[1,12]$. The representation of the risk zone is shown in Figure 2.

In the following we will describe the principle of the algorithm DVZ applied to the electric wheelchair. It develops in an unknown environment. It is surrounded by a deformable virtual zone; the geometry of this area depends on the linear and angular velocities and the wheelchair coordinates. The deformations of the risk zone are due to the interaction with the environment through the sensors. The calculation of the deformation measurements from the sensors is used to define the response of the platform.

3.1. The Undeformed Risk Zone. In order to obtain an analytic expression for the algorithm DVZ, it is considered as 
an elliptical shape risk zone. Either $P=\left[\begin{array}{ll}x & y\end{array}\right]^{T}$ a point on the ellipse with the axes $c_{x}$ and $c_{y}$. It is assumed that the reference position by the considered DVZ is translated from the center by a vector $a=\left[\begin{array}{ll}a_{x} & a_{y}\end{array}\right]^{T}$. Hence the equation is given by

$$
\left(\frac{x-a_{x}}{c_{x}}\right)^{2}+\left(\frac{y-a_{y}}{c_{y}}\right)^{2}=1 .
$$

Coefficients $a_{x}, a_{y}, c_{x}$, and $c_{y}$ are selected heuristically $[1,6]$. They depend on the speed of the wheelchair. We can modify the coefficients " $\sqrt{5} / 3$ " and " $-2 / 3$ " of the expression $c_{y}$ and $a_{x}$ according to the maximum speed of the wheelchair, the range sensors, and response time of the system

$$
\begin{aligned}
& c_{x}=\lambda_{c x} u^{2}+c_{x}^{\min }, \\
& c_{y}=\frac{\sqrt{5}}{3} c_{x}, \\
& a_{x}=\frac{-2}{3} c_{x}, \\
& a_{y}=0 .
\end{aligned}
$$

The distance $d_{h}$ is determined from the equality given by the following defined in $[1,6]$ :

$$
A d_{h}(\alpha)^{2}+B d_{h}(\alpha)+C=0
$$

Hence the expression $d_{h}$ is written as

$$
d_{h}(\alpha)=\frac{-B+\sqrt{(B)^{2}-4 A C}}{2 A},
$$

along with

$$
\begin{aligned}
& A=\left(c_{y} \cos (\alpha-\gamma)\right)^{2}+\left(c_{x} \sin (\alpha-\gamma)\right)^{2}, \\
& B=2 \cdot\left(a_{x} \cos (\alpha-\gamma) c_{y}^{2}+\left(a_{y} \sin (\alpha-\gamma) c_{x}^{2}\right)\right), \\
& C=\left(a_{x} c_{y}\right)^{2}+\left(a_{y} c_{x}\right)^{2}-\left(c_{x}^{2} c_{y}^{2}\right) ;
\end{aligned}
$$

$\gamma$ is the angle between axes $c_{x}$ and $X_{1}$. The risk zone is rigidly attached to the electric wheelchair and follows the position and direction during movement therefore $(\gamma=0)$. The detection range of the sensors used covers the front of the mobile platform.

3.2. The Deformed Risk Zone. The deformation of the area at risk is due to the existence of an obstacle. This deformation is calculated from the measurements of distance by the sensors. Distances measured by the ultrasonic sensors are rated $c(\alpha)$. The distance $d(\alpha)$ between the sensor and the risk zone is distorted by saturation obtained $c(\alpha)$ according to the follwoing algorithm:

$$
\begin{aligned}
d(\alpha) & =c(\alpha) \operatorname{sic}(\alpha)<d_{h}(\alpha) \\
& =d_{h}(\alpha) \text { sinon } .
\end{aligned}
$$

3.3. The Deformation. The intrusion report necessary for the development of response of the control algorithm upon detection of an obstacle is present. " $I$ " is the expression of intrusion given by equation report (10). When the distance between the robot and the obstacle approaches zero, it is found that " $I$ " tends to infinite. Then, a limitation was applied on the values of " $I$ " for a limited intrusion. This intrusion gives a good efficiency of obstacles avoidance system:

$$
I=\int_{\alpha=0}^{\pi} \frac{d_{h}(\alpha, u)-d(\alpha)}{d(\alpha)} d \alpha .
$$

The derivation of the expression of the intrusion versus time gives the following result [1]:

$$
\dot{I}=J_{I}^{u} \dot{u}+J_{I}^{\theta} r+F^{\text {Rob.vel }} u
$$

with

$$
F^{\text {Rob.vel }}=\int_{\alpha=0}^{\pi} \frac{d_{h}(\alpha)}{d^{2}(\alpha)} \cos (\alpha) d \alpha .
$$

The derived expressions are given in [12].

The inverse kinematic model is used to generate velocities necessary for controlling the two driving wheels of the electric wheelchair. The displacement allows obstacle avoidance. We denote the expression $V_{I}=I^{2} / 2$ which satisfies the Lyapunov stability theorem $[1,6]$. The equation of the derivative of $V_{I}$ relative to the intrusion " $I$ " gives

$$
\dot{V}_{I}=I\left(J_{I}^{u} \dot{u}+J_{I}^{\theta} r+F^{\text {Rob.vel }} u\right) .
$$

On our application, we will set the control system of the electric wheelchair given by [1]

$$
\begin{aligned}
& \dot{u}=-K_{u} J_{I}^{u} I-\frac{F^{\text {Rob.vel }}}{J_{I}^{u}} u, \\
& r=-K_{r} J_{I}^{\theta} I .
\end{aligned}
$$

Parameters $K_{u}$ and $K_{r}$ are positive gains resulting in $\dot{V}_{I} \leq$ $0 \forall t$.

3.4. Application of the DVZ Method for Obstacle Avoidance. We tested the algorithm of the obstacle avoidance using a developed simulator for electric wheelchair. The control parameters are selected according to constraints such as speed limits, the response time of the system, and the sampling period. The distances between obstacles and wheelchair are calculated in the simulation. We will discuss several cases in the simulation. In the following we will detail the simulation of the obstacle avoidance DVZ method algorithm. We will consider a case for the validation of the algorithm combining the fuzzy controller to reach a target point and obstacle avoidance. Then we will consider a complicated case with many obstacles.

(i) Figure 3 indicates a case of an obstacle avoidance. In this simulation the wheelchair must reach a target point with coordinates $\left(X_{T}=4 \mathrm{~m}, Y_{T}=4 \mathrm{~m}\right)$ with a 


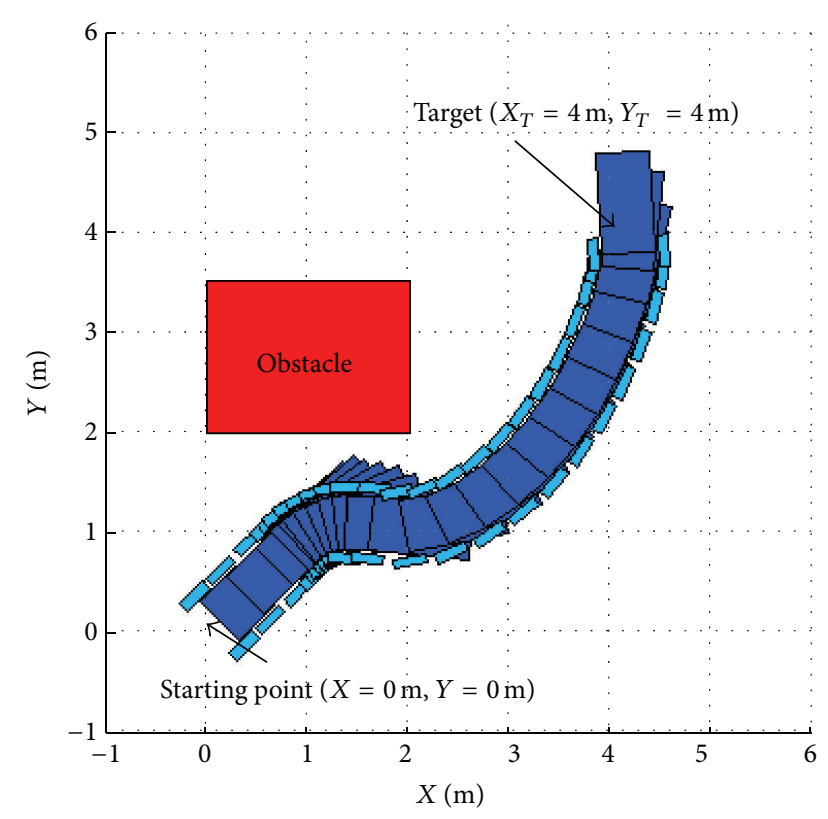

FIgURE 3: Application of the DVZ method.

starting point $(X=0 \mathrm{~m}, Y=0 \mathrm{~m})$ and a departure angle $\pi / 4$. The obstacle is in the middle of the path. The trajectory traveled by the electric wheelchair presented in Figure 3 is obtained by applying the fuzzy navigation controller combined with the DVZ method for obstacle avoidance.

(ii) In the last simulation, the navigation algorithm has provided obstacle avoidance using the left sensors. We will consider now a second simulation for complicated case. The navigation is performed in an environment containing a hallway and obstacles.

Figure 4 represents the case where the starting point of the wheelchair is $(X=-8 \mathrm{~m}, Y=4 \mathrm{~m})$ and a wheelchair orientation angle $\theta=0$. The target is $\left(X_{T}=5 \mathrm{~m}, Y_{T}=3 \mathrm{~m}\right)$. Figure 4 shows the trajectory with obstacle avoidance. As the tracking system is based on odometry information, the actual path may be different because of measurement errors and the statement of the initial position. The electric wheelchair detects and avoids obstacles without collision. Hysteresis function ensures switching between nonoptimized fuzzy controller to achieve the target location and the DVZ algorithm. We limit the wheel speeds during obstacle avoidance to ensure system stability during navigation.

In the next section, we will add a method of localization using particle filter to improve the localization and performance of the control algorithm.

\section{Particle Filter}

Particle filter, also known as sequential Monte Carlo (SMC) methods estimation techniques, is based on simulation. They are used in mobile robotics for localization, path planning, and vision [13]. In the following we will describe the use of

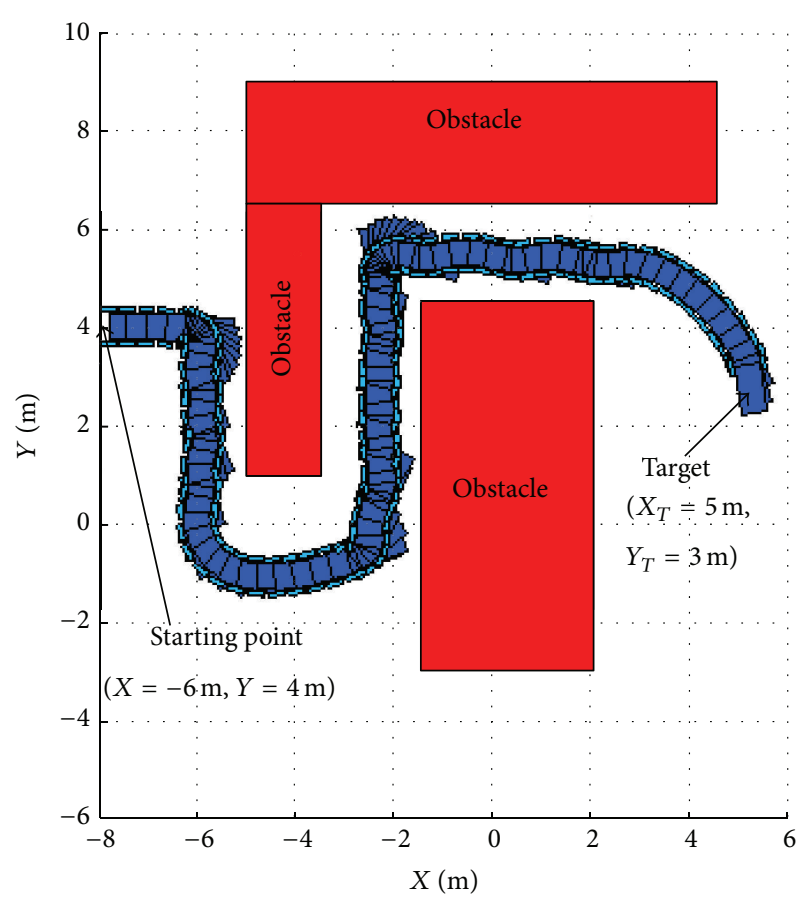

FIGURE 4: Wheelchair navigation in an environment with many obstacles.

the particle filter and fusion data used for locating an electric wheelchair for the disabled.

4.1. Particle Filter Algorithm for Locating an Electric Wheelchair. Particle filtering is a sequential version of the Monte Carlo methods to solve filtering problems. The conditional distribution of the state is represented by a finite number of weighted systems called Dirac particles. Each particle represents a state of probable weighted system. Its weight is based on the confidence level of the particle. It represents the probable state of the system. Historically the first filter contains a step redistribution presented as particle filter algorithm with interaction or condensation [4].

For the simulation, we use a discrete model defined by the system of (3).

Each particle is a configuration of the electric wheelchair. This particle is associated with a weight $p^{i}$ representing the consistency between the configuration of the particle and the configuration of the electric wheelchair in its environment from observations.

First Step (initialization of particle). Consider

$$
p^{i}=\left(x^{i}, w^{i}\right)
$$

where $w^{i}$ is associated with the weight of the $i$ particle configuration $x^{i}$ and $\varepsilon_{x}^{i}, \varepsilon_{y}^{i}, \varepsilon_{\theta}^{i}$ are random variables:

$$
P^{i}=\left(\left[\begin{array}{c}
x_{\text {init }}+\varepsilon_{x}^{i} \\
y_{\text {init }}+\varepsilon_{y}^{i} \\
\theta_{\text {init }}+\varepsilon_{\theta}^{i}
\end{array}\right], \frac{1}{N}\right) .
$$


Second Step (prediction). During this step, data encoders will be used to move the particles. The evolution of the particle is given by

$$
P_{k+1}^{i}=\left(\left[\begin{array}{c}
x_{k}^{i}+T \frac{V_{d k}+V_{g k}}{2} \cos \theta_{k} \\
y_{k}^{i}+T \frac{V_{d k}+V_{g k}}{2} \sin \theta_{k} \\
\theta_{k}^{i}+T \frac{V_{d k}-V_{g k}}{L}
\end{array}\right], w_{k}^{i}\right) .
$$

Step Three (estimation). In this step the information of the distance sensors is used. For each particle $i$ and $j$ for each sensor, we evaluate error $e_{y}^{i}$

$$
e_{y}^{i}=y^{j}-y_{m}^{j}\left(x^{i}\right)
$$

where $y^{j}$ is the distance given by $j$ th sensors and $y_{m}^{j}\left(x^{i}\right)$ is calculated by the developed simulator of sensors distance associated with the expression $x^{i}$.

We consider a Gaussian error $N\left(0, \sigma_{d}\right)$; that is to say, the probability of measuring $y^{j}$ configuration $x^{i}$ is given by

$$
p\left(y^{j} \mid x^{i}\right)=\exp ^{(-1 / 2)\left(\left(e_{y}^{i}\right)^{2} / \sigma_{y}^{2}\right)} \text {. }
$$

The new coefficient of the particle is calculated using the following relation:

$$
w_{k+1}^{i}=w_{k}^{i} \sum_{j=1}^{N} p\left(y^{j} \mid x^{i}\right) .
$$

The weight of the particles is adjusted using

$$
W^{i}=\frac{w^{i}}{\sum_{j=1}^{N} w^{j}} .
$$

Step Four (resampling). Resampling is performed when the inequality $N_{\text {eff }}<N_{\text {th }}$ is checked [7,14]. Initially, we create $\left[N w^{i}\right]$ copies particles $x^{i}$. [Nw $\left.w^{i}\right]$ is the integer part of $N w^{i}$. With this approximation, the number of generated particles is less than $N$. Therefore, it is necessary to add more particles observed by $\bar{N}$ and given by the following expression:

$$
\bar{N}=\sum_{i=1}^{N}\left(N w^{i}-\left[N w^{i}\right]\right) .
$$

Resampling is to generate multinomial $\bar{N}$ new particles. These particles are weighted by

$$
w^{i}=\frac{\left(N w^{i}-\left[N w^{i}\right]\right)}{\bar{N}} .
$$

In the next section we will describe the results obtained by combining the fuzzy controller, the DVZ method, and the particle filter for navigation and localization of electric wheelchair for disabled persons.

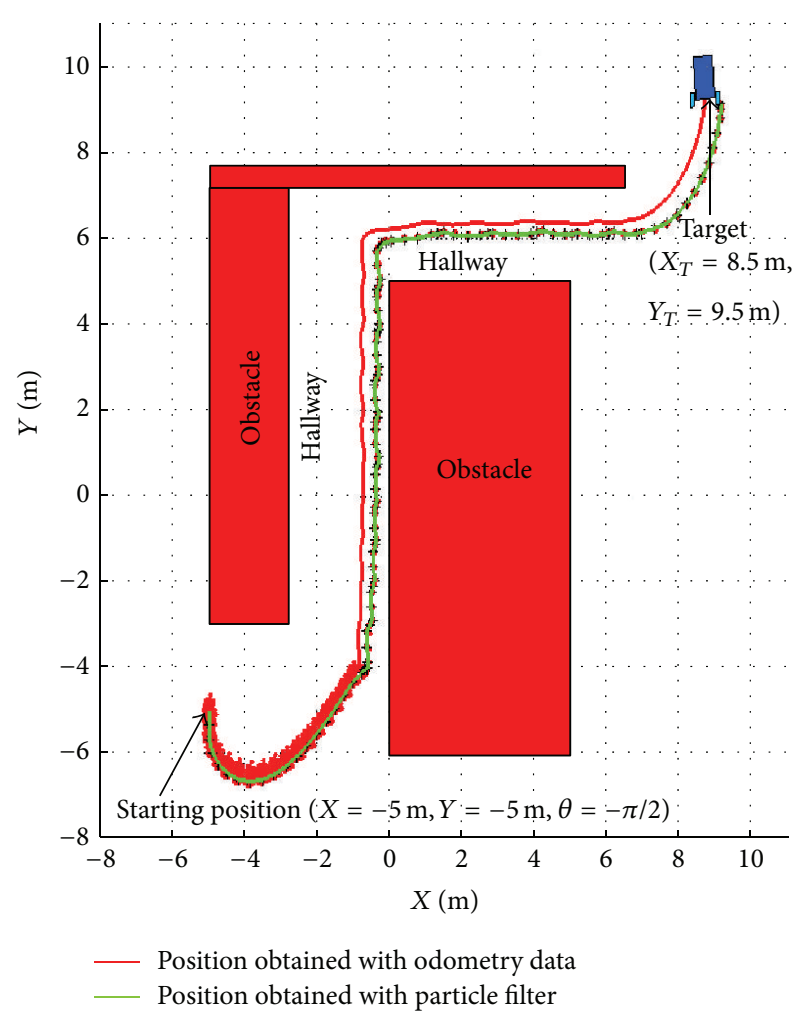

FIGURE 5: Simulation result of an electric wheelchair obtained by the combination of DVZ method, particle filter, and fuzzy controller.

\section{Result of the Electric Wheelchair Navigation}

The navigation of the electric wheelchair is provided by the fuzzy controller. The controller generates the wheels velocity to reach the target point. The DVZ method is used to avoid the obstacles in the way. The location of the electric wheelchair is calculated by the kinematic model by using the information received from encoders. We will introduce the localization using the particle filter. The sampling period used is $T=1$ second.

(i) Figure 5 shows the simulation result of a case of navigation with obstacle. The navigation environment presents obstacles to the right and left and a hallway.

The starting position is considered $(X=-5 \mathrm{~m}, Y=-5 \mathrm{~m}$, and $\theta=-\pi / 2)$ and the target position is $\left(X_{T}=8.5 \mathrm{~m}\right.$, $\left.Y_{T}=9.5 \mathrm{~m}\right)$. An error in the measurements of the sensors is added to test the localization algorithm by particle filter. These errors are due to the geometric errors parameters of the wheelchair (wheel diameter, length, and width), the slipping of wheels, errors of speed measurement, and errors in initial values of the orientation and position. The localization error increases when the wheelchair does not detect an obstacle. The localization with particle filter is used when the wheelchair avoids an obstacle with a known position. It is found that the algorithm of particle filter allows data fusion obtained by the simulation of the encoders and the distance measured by the distance sensors. Initially 1000 particles are generated. Particles evolved during navigation using (17). 
During that time detection of an obstacle, the particulate filter gives the estimated actual position and removes particles assuming a low weight. The particles removed are replaced by each other by the resampling. The navigation to achieve the target location is performed by the navigation fuzzy controller associated with the obstacle avoidance using DVZ method. The localization algorithm is using particle filter by using the kinematics model for the evolution of the position of the particles. The green curve is obtained by the particle filter path. This curve is the connection between the particles having the highest weight. The simulation result showed improved localization. The red curve (see Figure 5) gives the odometer position. The green curve obtained by fusion of the sensor data with the particulate filter shows better positioning of the wheelchair in its environment. The positioning error is considered in the simulation $400 \mathrm{~mm}$. In each position of the obstacle detection algorithm computes the actual position location.

The simulations obtained (see Figure 5) show considerable improvement in the precision of the localization by the particle filter. This method of localization will be used to reach a target location with better precision.

In the next section we will describe the proposed algorithm for applying the developed control system to a real wheelchair.

\section{Proposed Algorithm for Real Wheelchair Control}

In this section we present the proposed algorithm for application of the global control system. The algorithm used for real wheelchair is illustrated by Figure 6 .

(i) In the case of navigation without obstacle, the function "join target" is selected. The velocity generated by the fuzzy controller is applied on two driving wheels. This controller steers the robot to reach the target. After a sampling period, the measurement acquisition system sends the information given by the encoders and ultrasonic sensors. This information is necessary for localization and obstacle avoidance.

(ii) In the case when obstacle is in a risk zone, the condition $\left(d_{h}(\alpha)>d(\alpha)\right)$ is verified. " $d(\alpha)$ " is given by the measurement of each ultrasonic sensor $\left(D_{S 1}, D_{S 2}, D_{S 3}, D_{S 4}\right)$ and " $d_{h}(\alpha)$ " is calculated by the particle filter program. Each sensor admits an angle " $\alpha$ " and range and zone of detection. The "switch bloc" selects the algorithm for localization and obstacle avoidance. The distance obtained by the ultrasonic sensors and the theoretical distance are used by the particle filter for localization. After localization, the new position is $\left(X_{k}=X_{\mathrm{pf}}, Y_{k}=Y_{\mathrm{pf}}\right)$ with $\left(X_{\mathrm{pf}}, Y_{\mathrm{pf}}\right)$ the coordinate of particle having the most important weight calculated by (20). Next step is the obstacle avoidance using DVZ method. The DVZ function calculates the intrusion (equation (10)) and generates the linear and angular velocity using system of (14). We generate the velocity by the inverse kinematic

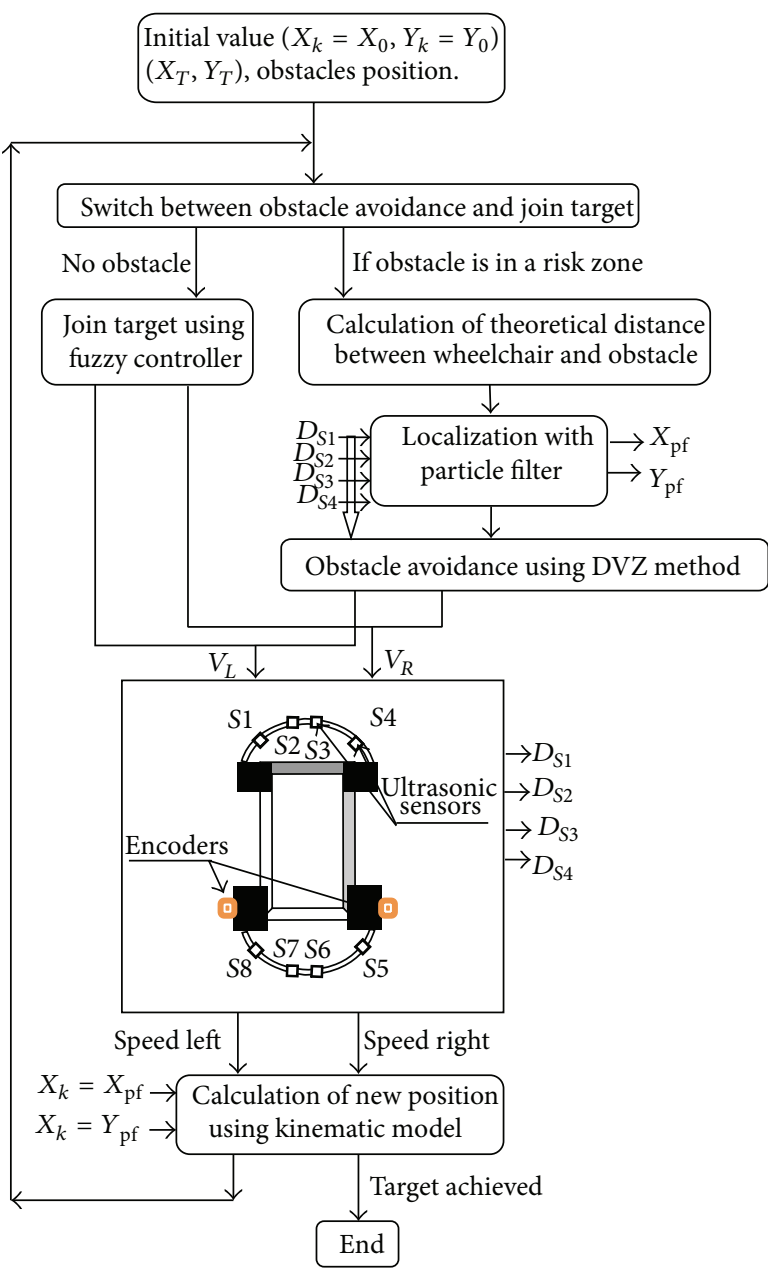

FIGURE 6: Proposed algorithm for real wheelchair control.

model. Then, the generated velocities are applied on two driving wheels.

(iii) If the wheelchair achieved the target the algorithm is finished.

\section{Conclusion}

In this paper we developed a control system for electric wheelchair navigation. The sensor data fusion is carried out by the particle filter for estimating the position of the electric wheelchair. It has been established with the kinematic model of the wheelchair with the fuzzy controller which achieves a target point. For the obstacle avoidance we used a DVZ method. With this control system we have improved the navigation precision of the wheelchair. The particle filter method in the navigation gives more details on the actual position and improves the quality of the control algorithm to achieve the target with minimum error. 


\section{Conflict of Interests}

The authors declare that there is no conflict of interests regarding the publication of this paper.

\section{References}

[1] L. Lapierre, R. Zapata, and P. Lepinay, "Combined path-following and obstacle avoidance control of a wheeled robot," The International Journal of Robotics Research, vol. 26, no. 4, pp. 361375, 2007.

[2] A. Gil Pinto, P. Fraisse, and R. Zapata, An Decentralized Adaptive Trajectory Planning Approach for a Group of Mobile Robots, LIRMM, Universit Montpellier II, 2006.

[3] A. Gil Pinto, P. Fraisse, and R. Zapata, "A decentralized algorithm to adaptive trajectory planning for a group of nonholonomic mobile robots," in Proceedings of the IEEE/RSJ International Conference on Intelligent Robots and Systems (IROS '06), pp. 404-409, Beijing, China, October 2006.

[4] F. Legland, "Filtrage particulaire," Project MathSTIC Chaînes de Markov Caches et Filtrage Particulaire, IRISA, Rennes, France, 2003.

[5] A. Kokosy, F.-O. Defaux, and W. Perruquetti, "Autonomous navigation of a nonholonomic mobile robot in a complex environment," in Proceedings of the IEEE International Workshop on Safety, Security and Rescue Robotics (SSRR '08), pp. 102-108, Sendai, Japan, November 2008.

[6] L. Lapierre, R. Zapata, and P. Lepinay, "Simulatneous path following and obstacle avoidance control of a unicycle-type robot," in Proceedings of the IEEE International Conference on Robotics and Automation, pp. 2617-2622, Roma, Italy, April 2007.

[7] J. S. Liu and R. Chen, "Sequential Monte Carlo methods for dynamic systems," Journal of the American Statistical Association, vol. 93, no. 443, pp. 1032-1044, 1998.

[8] M. Njah, M. Jallouli, and N. Derbel, "Optimal fuzzy controller for the navigation of an electric wheelchair," Transactions on Systems, Signals and Devices, vol. 6, 2011.

[9] M. Njah and M. Jallouli, "Synthesis of a fuzzy controller for the navigation of an electric wheelchair for handicapped," in Proceedings of the 6th IEEE International Multi-Conference on Systems, Signals and Devices (SSD '09), Djerba, Taiwan, March 2009.

[10] R. Zapata, P. Lépinay, and P. Thompson, "Reactive behaviors of fast mobile robots," Journal of Robotic Systems, vol. 11, no. 1, pp. 13-20, 1994.

[11] A. Cacitti and R. Zapata, "Reactive behaviours of mobile manipulators based on the DVZ approach," in Proceedings of the IEEE International Conference on Robotics and Automation (ICRA '01), pp. 680-685, May 2001.

[12] L. Lapierre and R. Zapata, "A guaranteed obstacle avoidance guidance system," Autonomous Robots, vol. 32, no. 3, pp. 177187, 2012.

[13] L. Brèthes, F. Lerasle, P. Danès, and M. Fontmarty, "Particle filtering strategies for data fusion dedicated to visual tracking from a mobile robot," Machine Vision and Applications, vol. 21, no. 4, pp. 427-448, 2010.

[14] A. Doucet, S. Godsill, and C. Andrieu, "On sequential Monte Carlo sampling methods for Bayesian filtering," Statistics and Computing, vol. 10, no. 3, pp. 197-208, 2000. 

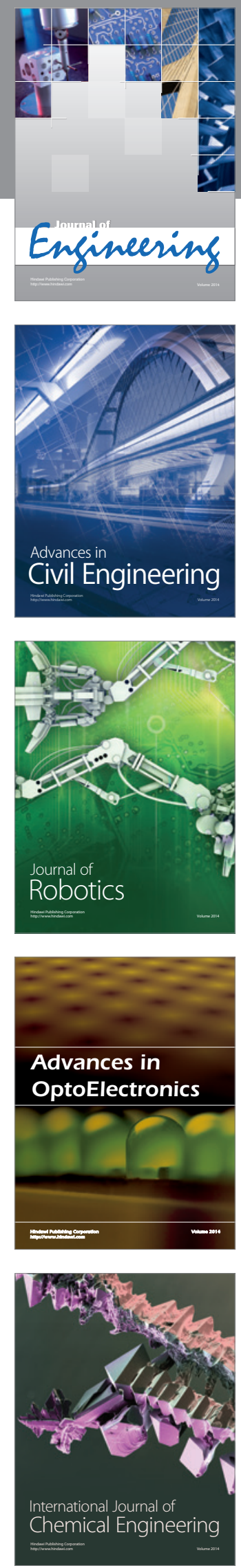

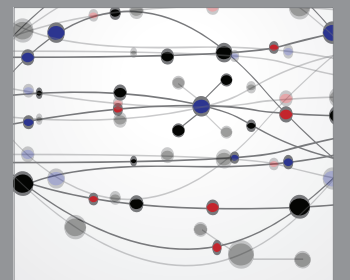

The Scientific World Journal
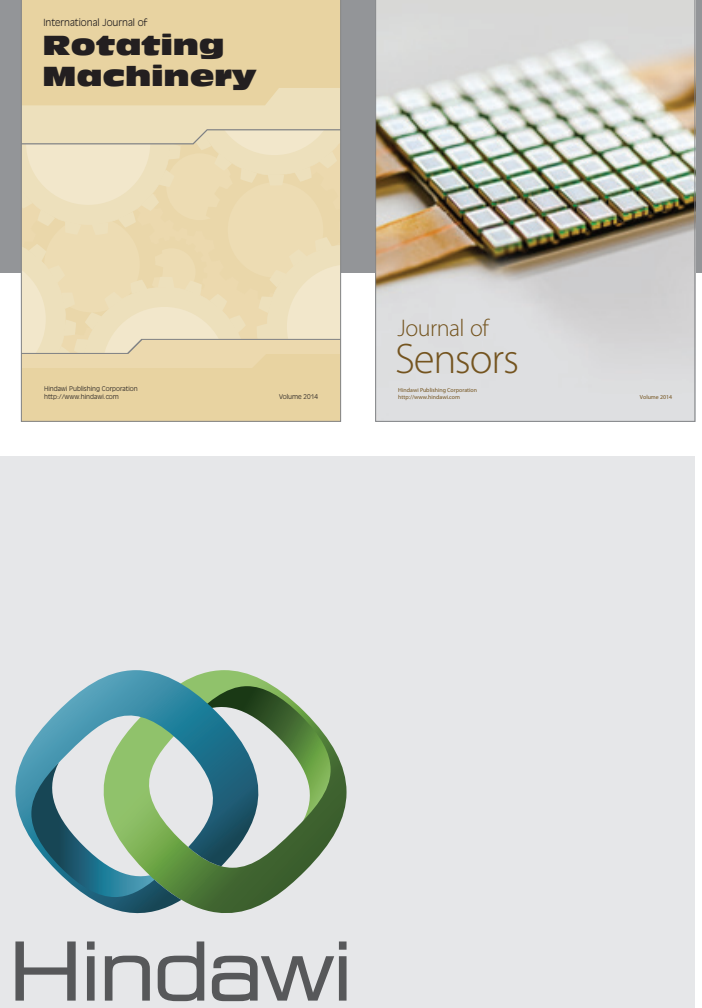

Submit your manuscripts at http://www.hindawi.com
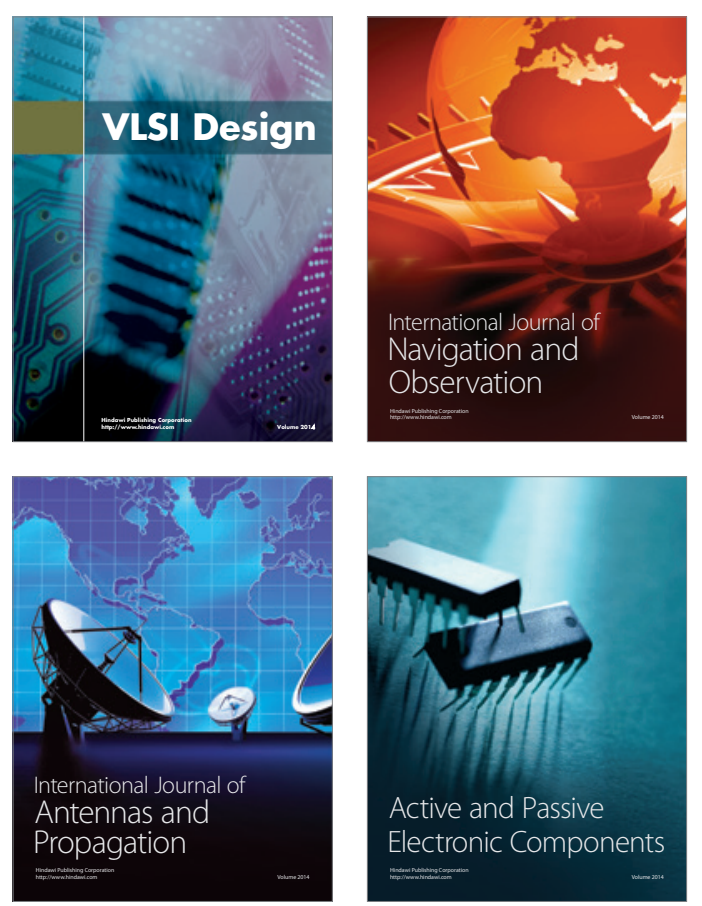
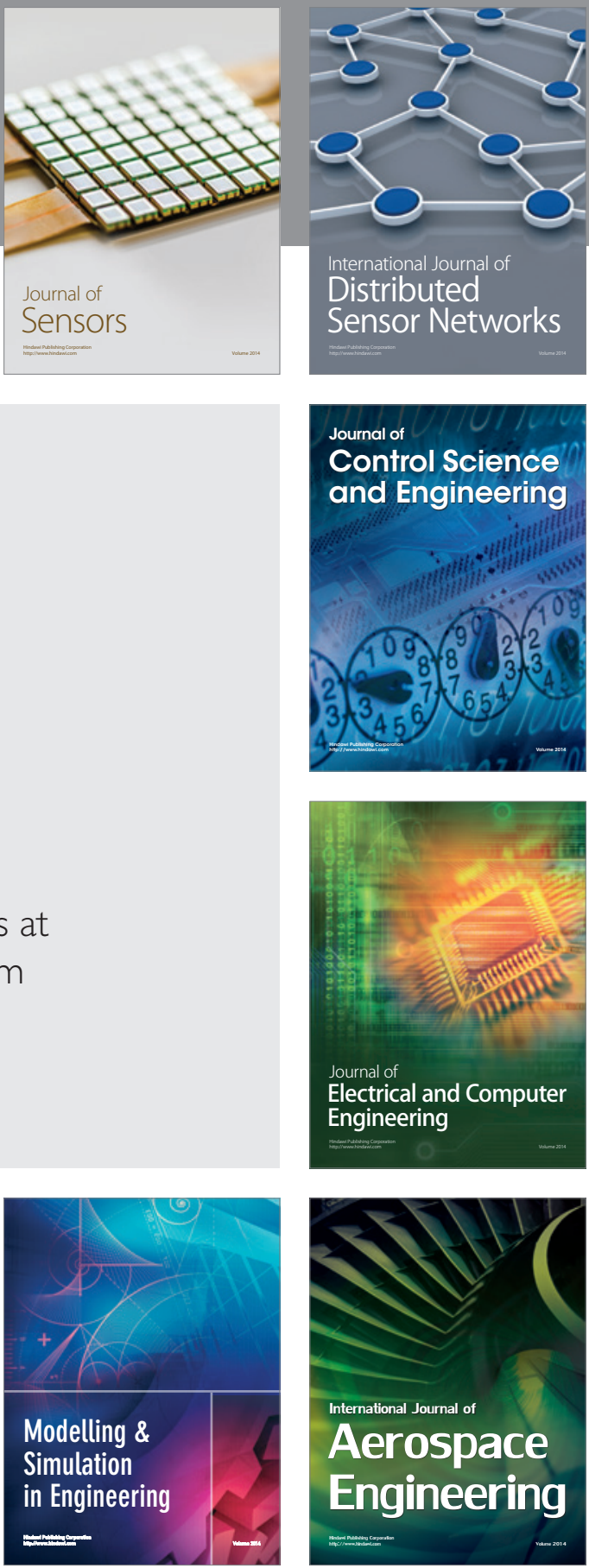

Journal of

Control Science

and Engineering
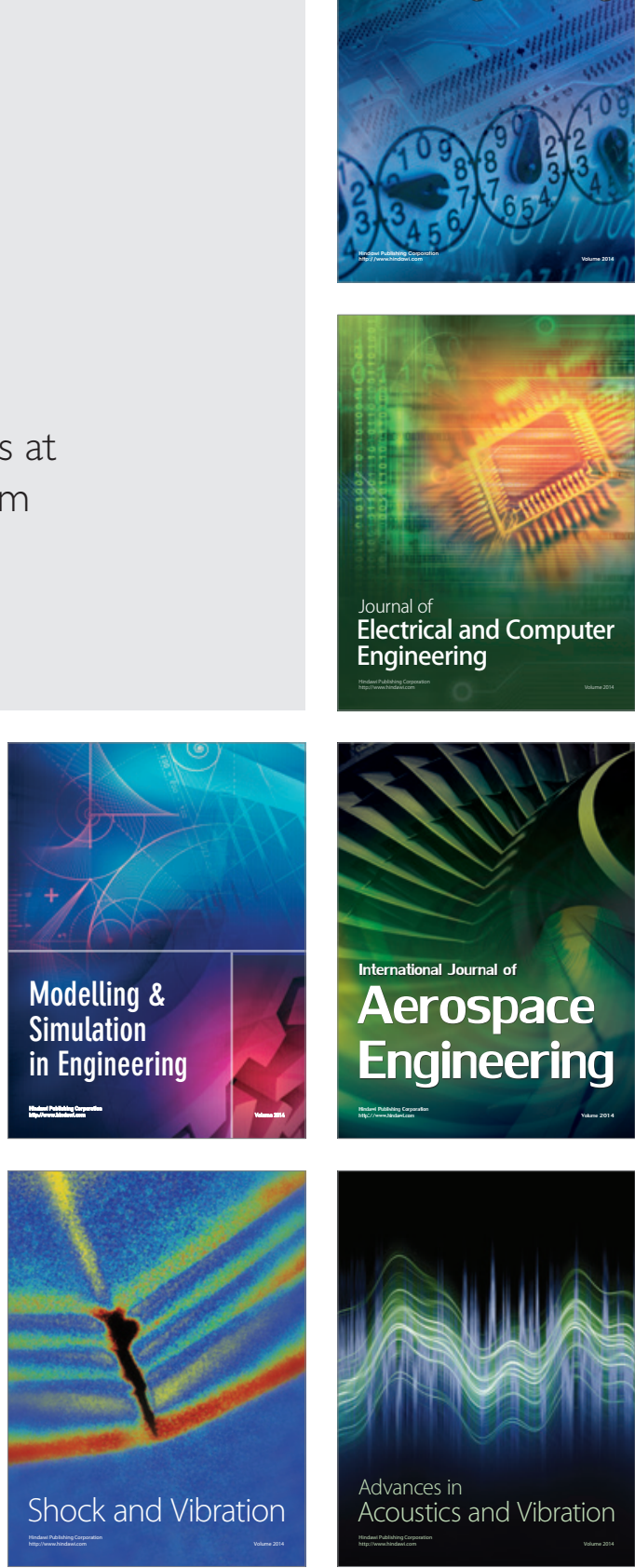